\title{
CHARGE-SYMMETRIC DESCRIPTION OF ELECTRONS AND POSITRONS IN THE DIRAC THEORY WITHOUT NEGATIVE ENERGIES
}

\author{
Yu. M. Poluektov* \\ National Science Center "Kharkiv Institute of Physics and Technology", 61108 Kharkiv, Ukraine
}

(Received May 7, 2019)

\begin{abstract}
A formulation of the Dirac theory, symmetric with respect to particles and antiparticles, in which negative energy states are excluded, is proposed. Fields of particles and antiparticles are associated with wave functions for which the Born interpretation as probability amplitudes is valid. Thus, in theory, various "paradoxes" are eliminated, the existence of which is due to incorrect accounting of states with negative energy.
\end{abstract}

PACS: 03.65.Pm, 14.60.Cd

\section{INTRODUCTION}

The relativistic equation for an electron was obtained by Dirac in his classical papers [1]. The exposition of Dirac's theory is available in many textbooks and monographs, some of which also became classical [215]. Like the non-relativistic Schrödinger equation, which describes the spatial and temporal evolution of a complex function, the Dirac equation also describes a complex function that includes four components. Solutions of the nonrelativistic Schrödinger equation for a free particle with positive energy form a complete set of states, according to which an arbitrary solution can be decomposed. In the Dirac theory, along with solutions with positive energy, there are solutions with the opposite sign of energy. Soon after the appearance of the work of Dirac, Schrödinger proposed simply to exclude from consideration the states with negative energy and give physical meaning only to states with positive energy [9]. However, as it turned out, this can not be done. In contrast to the nonrelativistic theory, solutions of the Dirac equation corresponding to states with positive energy do not form a complete set of states, and to obtain a general solution, solutions with negative energy should also be taken into account. In this regard, after it became clear that it was impossible to exclude negative energies from the theory, a problem arose with the physical interpretation of such solutions [1]. To this purpose, Dirac suggested that in nature almost all states with negative energy are occupied, and unoccupied states (holes) behave like positively charged particles. At first, Dirac identified such states with protons, but from symmetry considerations it followed that the mass of electrons and holes should be the same. The result of the realization of this circumstance was the hypothesis that new particles correspond to the holes, then unknown to science, with the same mass as that of an electron, but with the opposite sign of charge. Dirac's theory describes both positively and negatively charged particles with equal rights, while the "electronic sea" hypothesis breaks the symmetry between them. The interpretation of Dirac, which in itself does not follow from the mathematical formalism of his theory, was rather coldly accepted by many leading physicists. Only the discovery of the positron changed her attitude. Meanwhile, the experimental confirmation of some predictions of the theory does not make the theory itself logically flawless. Although over time it became clear that the Dirac theory for its interpretation does not need additional constructions, the interpretation at the "particle-hole" level is reproduced in many books on relativistic quantum mechanics and field theory [6]. At present, there is a completely consistent interpretation of Dirac's theory, which does not attract additional, intrinsically non-peculiar, qualitative considerations [2], although, in our opinion, this correct point of view is not always consistently carried out.

When interpreting the theory, the central question is: "What is the physical meaning of the complex multicomponent field, which is described by the Dirac equation?". This question is not purely speculative, but is of practical importance, since, depending on the answer to it, the rules for calculating the observed values should be established. If the Schrödinger complex field, according to the Born interpretation, is the probability amplitude, and its phase-invariant combinations correspond to the probability density and the probability flow density, then the general solution of the Dirac equation containing the contribution of states with both positive and negative energies does not allow such interpretation. Since such a general solution of the Dirac equation does not make sense

\footnotetext{
*Corresponding author E-mail address: yuripoluektov@kipt.kharkov.ua
} 
of the probability amplitude, it cannot be used to calculate average values of observed values and other probability characteristics. The usual probabilistic interpretation is allowed only by solutions with positive energies, through which the general solution of the Dirac equation can be expressed. However, this general solution is not a new admissible state in accordance with the quantum principle of superposition, since it involves the operation of charge conjugation. Oblivion of this circumstance leads to the emergence of various "paradoxes", such as the "Klein paradoxes" $[2,6]$, as well as to such phenomena in theory as the "jitter" of the electron $[6,13]$.

This paper proposes a variant of the Dirac theory in which equations for particles with opposite charge signs and equal masses are simultaneously and symmetrically considered, and where the independent variables describing physically realizable states are solutions for particles with opposite charge signs and positive energies. Solutions of equations that correspond to negative energies are not independent, but are expressed in terms of solutions with positive energies using the charge conjugation operation. This eliminates the problem of negative energies in theory. Complex wave functions describing the states of a particle and antiparticles admit the usual quantum mechanical interpretation and have the meaning of probability amplitudes. The equations for particles and antiparticles are independent, so they should be considered separately.

\section{CHARGE-SYMMETRIC FORM DIRAC EQUATIONS}

The Dirac equation describes the evolution of a fourcomponent complex function $\psi(x)=\psi_{\sigma}(\mathbf{x}, t)$, where index takes values $\sigma=1,2,3,4$. In the matrix notation, the Dirac equation for the function $\psi(x)$ and the conjugate function $\bar{\psi}(x)=\psi^{+} \gamma_{4}$ are

$$
\begin{aligned}
& \hbar c \gamma_{\mu} \frac{\partial \psi(x)}{\partial x_{\mu}}+m c^{2} \psi(x)=0, \\
& \hbar c \frac{\partial \bar{\psi}(x)}{\partial x_{\mu}} \gamma_{\mu}-m c^{2} \psi(x)=0,
\end{aligned}
$$

where $x \equiv x_{\mu}=\left(\vec{x}, x_{4}\right)=\left(\vec{x}, i x_{0}\right)=(\vec{x}, i c t), m-$ particle mass, $c$ - the speed of light, $\hbar$ - Planck's constant, $\gamma_{\mu}-$ Hermitian $4 \times 4-$ Dirac matrices. The sign + in $\psi^{+}(x)$ means Hermitian conjugation. Over repeated indices everywhere means summation. We mainly adhere to the notation of the book [2]. Accounting for interaction with the electromagnetic field is carried out using a well-known replacement

$$
\frac{\partial}{\partial x_{\mu}} \rightarrow \frac{\partial}{\partial x_{\mu}}-\frac{i e}{\hbar c} A_{\mu}(x),
$$

where $A_{\mu}(x)$ - four-vector potential, and charge $e= \pm|e|$ or $e=0$ for electrically neutral particles. In the following, we consider charged particles. Usually, when writing the Dirac equation, in (2) choose the electron charge. This initially introduces some asymmetry into the theory. However, since oppositely charged particles enter the theory perfectly symmetrical, for a charge-symmetric consideration it is convenient to introduce two fields of particles with the same masses, but opposite signs of charge - a "negative" field $\psi$, which we will consider the field of particles, and a "positive" field $\eta$, which we call the field antiparticles. It is accepted to consider a particle with a negative charge $e=-|e|$ (electron) as a "particle" and a particle with a positive charge $e=|e|$ (positron) as an "antiparticle". We do not discuss the asymmetry observed in nature between particles and antiparticles, which, quite likely, is not associated with a charge symmetry breaking in fundamental equations. Note also that the state of a particle can additionally be characterized by an "internal" (lepton) quantum number $\Lambda$, which for the particle is assumed positive, then the antiparticle is characterized by a lepton quantum number of opposite sign. The presence of the "internal" quantum number allows us to distinguish the fields of particles and antiparticles in the absence of an electromagnetic field, as well as in the case of neutral particles. Thus, the Dirac equations for the fields of charged particles and antiparticles in an electromagnetic field $A_{\mu}=\left(\vec{A}, i A_{0}\right)$ take the form

$$
\begin{aligned}
& \text { a) } \quad \hbar c \gamma_{\mu}\left(\frac{\partial \psi_{\Lambda}}{\partial x_{\mu}}-\frac{i e}{\hbar c} A_{\mu} \psi_{\Lambda}\right)+m c^{2} \psi_{\Lambda}=0 \\
& \text { b) } \quad \hbar c \gamma_{\mu}\left(\frac{\partial \eta_{-\Lambda}}{\partial x_{\mu}}+\frac{i e}{\hbar c} A_{\mu} \eta_{-\Lambda}\right)+m c^{2} \eta_{-\Lambda}=0
\end{aligned}
$$

In the future, the index of the internal quantum number $\Lambda$ for brevity will be omitted everywhere. Functions $\psi$ and $\eta$ are not independent. Their relationship can be established using a unitary charge conjugation matrix, which satisfies the conditions [2]:

$$
C^{+} C=C C^{+}=1, \quad C \gamma_{\mu} C^{+}=-\tilde{\gamma}_{\mu}, \quad C=-\tilde{C} .
$$

The sign means transpose. This matrix can be selected as $C=\gamma_{2} \gamma_{4}$. Then, from equations (3) and (4), the following relations follow:

$$
\text { a) } \psi=C^{*} \tilde{\bar{\eta}}, \quad \text { b) } \quad \eta=C^{*} \tilde{\bar{\psi}},
$$

binding solutions of Dirac equations with opposite charge signs.

Expand the solution of the Dirac equation into the Fourier integral:

$$
\psi(x)=\int_{-\infty}^{\infty} c(\omega) \psi(\vec{x}, \omega) e^{-i \omega t} d \omega=\psi_{+}(x)+\psi_{-}(x),
$$

where the positive-frequency and negative-frequency functions are defined by the formulas:

$$
\psi_{ \pm}(x)=\int_{0}^{\infty} c( \pm \omega) \psi(\vec{x}, \pm \omega) e^{\mp i \omega t} d \omega .
$$

It is assumed that there is an integral

$$
\frac{1}{2 \pi} \int_{-\infty}^{\infty} \psi(x) e^{i \omega t} d t<\infty
$$


Note that the integration in (7) is performed only by positive frequencies. Similarly, the solution of an equation with the opposite sign of charge is represented:

$$
\eta(x)=\int_{-\infty}^{\infty} b(\omega) \eta(\vec{x}, \omega) e^{-i \omega t} d \omega=\eta_{+}(x)+\eta_{-}(x),
$$

where

$$
\eta_{ \pm}(x)=\int_{0}^{\infty} b( \pm \omega) \eta(\vec{x}, \pm \omega) e^{\mp i \omega t} d \omega .
$$

Then from formulas (5) follow the relations expressing negative frequency functions in terms of positive frequency functions:

$$
\begin{array}{ll}
\psi_{-}(x)=C^{*} \tilde{\bar{\eta}}_{+}(x), & \bar{\psi}_{-}(x)=\tilde{\eta}_{+}(x) C, \\
\eta_{-}(x)=C^{*} \tilde{\bar{\psi}}_{+}(x), & \bar{\eta}_{-}(x)=\tilde{\psi}_{+}(x) C .
\end{array}
$$

Thus, general solutions of the Dirac equation with a charge of an arbitrary sign can only be expressed in terms of positive-frequency partial solutions $\psi_{+}(x)$ and $\eta_{+}(x)$ for Dirac equations with opposite charge signs and equal masses, which should be regarded as particle and antiparticle wave functions that allow Born probabilistic interpretation:

$$
\begin{gathered}
\psi(x)=\psi_{+}(x)+C^{*} \tilde{\bar{\eta}}_{+}(x), \\
\eta(x)=\eta_{+}(x)+C^{*} \tilde{\bar{\psi}}_{+}(x) .
\end{gathered}
$$

Since the positive-frequency particular solutions are interpreted as wave functions of the particle and antiparticle, having a meaning of probability amplitudes, they must be normalized by the conditions:

$$
\int\left|\psi_{+}(x)\right|^{2} d^{3} x=1, \quad \int\left|\eta_{+}(x)\right|^{2} d^{3} x=1 .
$$

General solutions of the Dirac equation (12) are expressed both through the wave function of the particle and the wave function of the antiparticle, but they are not a linear superposition of these functions, because they contain an antilinear transformation of complex conjugation, and therefore have no sense of the probability amplitudes. Thus, functions (12) containing the contribution of states with negative energies are not physically realizable states that have a meaning of probability amplitudes, and, therefore, they cannot be used when calculating transition probabilities and average values of observable operators. It is the illegal use in the calculations of such functions, including states with negative energy, that leads to the appearance in theory of various "paradoxes".

\section{DIRAC EQUATIONS FOR PROBABILITY AMPLITUDES}

Substitute the functions (12) into equations (3) and (4). As a result, we arrive at equations containing only functions with positive frequencies, which can be written as

$$
\begin{array}{ll}
Q(x)+C^{*} \tilde{\bar{\Pi}}(x)=0, & \bar{Q}(x)+\tilde{\Pi}(x) C=0, \\
\Pi(x)+C^{*} \tilde{\bar{Q}}(x)=0, & \bar{\Pi}(x)+\tilde{Q}(x) C=0,
\end{array}
$$

where designations are used

$$
\begin{gathered}
Q(x) \equiv \hbar c \gamma_{\mu} \frac{\partial \psi_{+}}{\partial x_{\mu}}-i e A_{\mu} \gamma_{\mu} \psi_{+}+m c^{2} \psi_{+}, \\
\Pi(x) \equiv \hbar c \gamma_{\mu} \frac{\partial \eta_{+}}{\partial x_{\mu}}+i e A_{\mu} \gamma_{\mu} \eta_{+}+m c^{2} \eta_{+},
\end{gathered}
$$

and $\bar{Q}(x) \equiv Q^{+}(x) \gamma_{4}, \bar{\Pi}(x) \equiv \Pi^{+}(x) \gamma_{4}$. Any three equations (14) are a consequence of the fourth equation, so relations (14) give a different form of writing one equation. We will first basically consider the case of a stationary electromagnetic field, assuming $A_{\mu}(x)=A_{\mu}(\mathbf{x})$. Using expansions (7) and (10) for functions $\psi_{+}(x)$ and $\eta_{+}(x)$, we find

$$
\begin{aligned}
& Q(x)=\int_{0}^{\infty} c(\omega) Q(\vec{x}, \omega) e^{-i \omega t} d \omega, \\
& \Pi(x)=\int_{0}^{\infty} b(\omega) \Pi(\vec{x}, \omega) e^{-i \omega t} d \omega,
\end{aligned}
$$

where

$$
\begin{gathered}
Q(\vec{x}, \omega)= \\
=\left[\hbar c\left(\vec{\gamma} \nabla-\frac{\omega}{c} \gamma_{4}\right)+m c^{2}-i e A_{\mu}(\vec{x}) \gamma_{\mu}\right] \psi(\vec{x}, \omega), \\
\Pi(\vec{x}, \omega)= \\
=\left[\hbar c\left(\vec{\gamma} \nabla-\frac{\omega}{c} \gamma_{4}\right)+m c^{2}+i e A_{\mu}(\vec{x}) \gamma_{\mu}\right] \eta(\vec{x}, \omega) .
\end{gathered}
$$

In this notation, equations (14) are equivalent to

$$
\begin{aligned}
& \int_{0}^{\infty}\left[c(\omega) Q(\vec{x}, \omega) e^{-i \omega t}+\right. \\
& \left.+b^{*}(\omega) C^{*} \tilde{\bar{\Pi}}(\vec{x}, \omega) e^{i \omega t}\right] d \omega=0 .
\end{aligned}
$$

Multiplying (18) first by $e^{i \omega^{\prime} t}$, where $\omega^{\prime}>0$, and integrating over time, and then multiplying by $e^{-i \omega^{\prime} t}$, and also performing integration over time, we get what should be $Q(\vec{x}, \omega)=0$ and $\tilde{\bar{\Pi}}(\vec{x}, \omega)=0$. Thus, we have arrived at two independent equations for the particle and antiparticle wave functions, which, given the notation (17), have the form

$$
\begin{aligned}
& {\left[\hbar c \vec{\gamma}\left(\nabla-i \frac{e}{\hbar c} \vec{A}(\vec{x})\right)-\right.}\left(\hbar \omega-e A_{0}(\vec{x})\right) \gamma_{4}+ \\
&+\left.m c^{2}\right] \psi(\vec{x}, \omega)=0, \\
& {\left[\hbar c \vec{\gamma}\left(\nabla+i \frac{e}{\hbar c} \vec{A}(\vec{x})\right)-\right.}\left(\hbar \omega+e A_{0}(\vec{x})\right) \gamma_{4}+ \\
&\left.+m c^{2}\right] \eta(\vec{x}, \omega)=0 .
\end{aligned}
$$

Here the functions $\psi(\vec{x}, \omega)$ and $\eta(\vec{x}, \omega)$, according to (7) and (10), depend only on the positive frequency. From equations (19), (20) the orthonormal conditions for electron and positron wave functions follow:

$$
\begin{aligned}
\int d^{3} x \psi^{+}(\vec{x}, \omega) \psi\left(\vec{x}, \omega^{\prime}\right) & =\delta\left(\omega-\omega^{\prime}\right), \\
\int d^{3} x \eta^{+}(\vec{x}, \omega) \eta\left(\vec{x}, \omega^{\prime}\right) & =\delta\left(\omega-\omega^{\prime}\right) .
\end{aligned}
$$


The conditions of orthogonality of the electronic and positron functions are also satisfied:

$$
\begin{aligned}
\int \psi^{+}\left(\vec{x}, \omega^{\prime}\right) \gamma_{4} C^{*} \eta^{*}(\vec{x}, \omega) d^{3} x & =0, \\
\int \tilde{\eta}(\vec{x}, \omega) C \gamma_{4} \psi\left(\vec{x}, \omega^{\prime}\right) d^{3} x & =0 .
\end{aligned}
$$

Conditions

$$
\int|c(\omega)|^{2} d \omega=1, \quad \int|b(\omega)|^{2} d \omega=1,
$$

decomposition coefficients (7), (10) and relations (21) provide the normalization conditions (13), and relations (22) lead to the conditions of the orthogonality of the functions of states with positive and negative energies:

$$
\int \psi_{+}^{+}(x) \psi_{-}(x) d^{3} x=0, \quad \int \eta_{+}^{+}(x) \eta_{-}(x) d^{3} x=0 .
$$

For time-dependent wave functions, the equations for particles and antiparticles have the form of the Dirac equations, differing only in the charge sign:

$$
\begin{array}{r}
\hbar c \gamma_{\mu}\left(\frac{\partial \psi_{+}(x)}{\partial x_{\mu}}-i \frac{e}{\hbar c} A_{\mu}(\vec{x}) \psi_{+}(x)\right)+ \\
+m c^{2} \psi_{+}(x)=0 \\
\hbar c \gamma_{\mu}\left(\frac{\partial \eta_{+}(x)}{\partial x_{\mu}}+i \frac{e}{\hbar c} A_{\mu}(\vec{x}) \eta_{+}(x)\right)+ \\
+m c^{2} \eta_{+}(x)=0 .
\end{array}
$$

Thus, in stationary fields, the equations for the wave functions of particles and antiparticles with positive energies are uncoupled, and, therefore, the states of particles and antiparticles can be considered independently.

\section{LAGRANGIAN FORMALISM}

We formulate a developed approach to the description of particles and antiparticles in terms of probability amplitudes, using the Lagrangian formalism, which allows us to obtain the energy-momentum tensor and conservation laws. The Dirac equations (25) can be obtained if the density of the Lagrange function is represented as a sum of Lagrangians particles $\Lambda_{\psi}(e)$ and $\Lambda_{\eta}(-e)$ antiparticles :

$$
\begin{array}{r}
\Lambda_{\psi}(e)=-\frac{c \hbar}{2}\left(\bar{\psi}_{+} \gamma_{\mu} \frac{\partial \psi_{+}}{\partial x_{\mu}}-\frac{\partial \bar{\psi}_{+}}{\partial x_{\mu}} \gamma_{\mu} \psi_{+}\right)+ \\
+i e A_{\mu}(\vec{x}) \bar{\psi}_{+} \gamma_{\mu} \psi_{+}-m c^{2} \bar{\psi}_{+} \psi_{+}, \\
\Lambda_{\eta}(-e)=-\frac{c \hbar}{2}\left(\bar{\eta}_{+} \gamma_{\mu} \frac{\partial \eta_{+}}{\partial x_{\mu}}-\frac{\partial \bar{\eta}_{+}}{\partial x_{\mu}} \gamma_{\mu} \eta_{+}\right)- \\
-i e A_{\mu}(\vec{x}) \bar{\eta}_{+} \gamma_{\mu} \eta_{+}-m c^{2} \bar{\eta}_{+} \eta_{+} .
\end{array}
$$

Independent dynamic variables are functions $\psi_{+}, \overline{\psi_{+}}$ and $\eta_{+}, \overline{\eta_{+}}$. Since, as was shown, in a stationary field, particles and antiparticles are described independently, it is sufficient to consider the case of particles, then similar relations for antiparticles will be obtained if we replace $e \rightarrow-e$ and $\psi_{+} \rightarrow \eta_{+}$. From the Euler-Lagrange equations, taking into account the form of the Lagrangians (26), (27), equations (25) follow, which were obtained directly from the Dirac equation above. From the condition of invariance of the Lagrangian (26) for a particle with respect to phase transformations:

$$
\begin{gathered}
\psi_{+}(x) \rightarrow \psi_{+}^{\prime}(x)=\psi_{+}(x) e^{i \alpha}, \\
\bar{\psi}_{+}(x) \rightarrow \bar{\psi}_{+}^{\prime}(x)=\bar{\psi}_{+}(x) e^{-i \alpha},
\end{gathered}
$$

where $\alpha$ is the real parameter, the continuity equation for the probability density of the particle follows

$$
\frac{\partial j_{\psi \mu}}{\partial x_{\mu}}=0
$$

where the 4-vector probability density flow has the form

$$
j_{\psi \mu}=i c \bar{\psi}_{+} \gamma_{\mu} \psi_{+} .
$$

From (29) follows the law of conservation of the total probability for a particle $\int d^{3} x j_{\psi_{0}}(x)=$ const. These relations are similar to those in the nonrelativistic quantum theory [16]. The densities of the Lagrange functions (26), (27) depend on the wave functions of the particle and the antiparticle and on the electromagnetic field, which is considered as an external field, but they do not explicitly depend on $x$. Because of this, the form of the Lagrangians should not change when the entire system, including the external field, is translated into an arbitrary 4-vector $\alpha_{\mu}$. From these considerations, we find the equation for the energy-momentum tensor:

$$
\frac{\partial T_{\psi \mu \nu}}{\partial x_{\nu}}=-\frac{\partial \Lambda_{\psi}}{\partial A_{\nu}(x)} \frac{\partial A_{\nu}(x)}{\partial x_{\mu}},
$$

where the energy-momentum tensor related to a particle is defined by the well-known relation

$$
T_{\psi \mu \nu}=\frac{\partial \Lambda_{\psi}}{\partial \frac{\partial \psi_{+}}{\partial x_{\nu}}} \frac{\partial \psi_{+}}{\partial x_{\mu}}+\frac{\partial \bar{\psi}_{+}}{\partial x_{\mu}} \frac{\partial \Lambda_{\psi}}{\partial \frac{\partial \bar{\psi}_{+}}{\partial x_{\nu}}}-\Lambda_{\psi} \delta_{\mu \nu} .
$$

It is customary to introduce a 4 -vector momentum

$$
P_{\psi \mu}=\frac{i}{c} \int T_{\psi \mu 4} d \mathbf{x}
$$

at that $P_{\psi \mu} \equiv\left(\vec{P}_{\psi}, \frac{i}{c} W_{\psi}\right)$. Total momentum $\vec{P}_{\psi}$ and total energy $W_{\psi}$ are determined by the formulas

$$
P_{\psi i}=\frac{i}{c} \int T_{\psi i 4} d^{3} x, \quad W_{\psi}=\int T_{\psi 44} d^{3} x .
$$

Taking into account the form of the Lagrangian (26), and also the fact that for functions satisfying the Dirac equation, the Lagrangian (26) vanishes, we find the energy-momentum tensor expressed through the wave functions of a particle with positive energy:

$$
T_{\psi \mu \nu}=-\frac{c \hbar}{2} \bar{\psi}_{+} \gamma_{\nu} \frac{\partial \psi_{+}}{\partial x_{\mu}}+\frac{c \hbar}{2} \frac{\partial \bar{\psi}_{+}}{\partial x_{\mu}} \gamma_{\nu} \psi_{+} .
$$


Taking into account the law of conservation of probability (29), the energy-momentum 4-vector can be represented as

$$
P_{\psi \mu}=-i \hbar \int \psi_{+}^{+} \frac{\partial \psi_{+}}{\partial x_{\mu}} d^{3} x
$$

so the total momentum $\mathbf{P}_{\psi}$ and particle energy $W_{\psi}=$ $-i c P_{\psi 4}$ are given by the ratios

$$
\begin{gathered}
\vec{P}_{\psi}=-i \hbar \int \psi_{+}^{+} \nabla \psi^{+} d^{3} x \\
W_{\psi}=i \hbar \int \psi_{+}^{+} \frac{\partial \psi_{+}}{\partial t} d^{3} x .
\end{gathered}
$$

These formulas are similar to the formulas for calculating the mean momentum and mean energy in nonrelativistic quantum mechanics. A specific feature of the field relations obtained here is that the fields in them have the meaning of complex probability amplitudes for particles and antiparticles, and the time dependence of the fields is determined by Fourier by expansions $(7),(10)$ only by positive frequencies.

\section{FREE PARTICLES AND ANTI-PARTICLES}

Let us apply the proposed interpretation of the Dirac theory to the description of free particles and antiparticles. In the absence of an external field, the equations for a particle and an antiparticle are the same:

$$
\begin{gathered}
{\left[\hbar c \vec{\gamma} \nabla-E \gamma_{4}+m c^{2}\right] \psi_{+}(\vec{x}, E)=0,} \\
{\left[\hbar c \vec{\gamma} \nabla-E \gamma_{4}+m c^{2}\right] \eta_{+}(\vec{x}, E)=0,}
\end{gathered}
$$

where the designation for positive energy $E \equiv \hbar \omega$ is entered. We look for solutions of these equations in the form of plane waves

$$
\begin{aligned}
\psi_{+}(\vec{x}, E) & =\frac{1}{\sqrt{V}} \psi(\vec{k}) e^{i \vec{k} \vec{x}} \\
\eta_{+}(\vec{x}, E) & =\frac{1}{\sqrt{V}} \eta(\vec{k}) e^{i \vec{k} \vec{x}}
\end{aligned}
$$

In this instance

$$
\begin{gathered}
{\left[i \hbar c \vec{k} \vec{\gamma}-E \gamma_{4}+m c^{2}\right] \psi(\vec{k})=0,} \\
{\left[i \hbar c \vec{k} \vec{\gamma}-E \gamma_{4}+m c^{2}\right] \eta(\vec{k})=0 .}
\end{gathered}
$$

Bispinores can be written as columns of spinors

$$
\psi(\vec{k})=\left[\begin{array}{c}
\varphi(\vec{k}) \\
\chi(\vec{k})
\end{array}\right], \quad \eta(\vec{k})=\left[\begin{array}{c}
\zeta(\vec{k}) \\
v(\vec{k})
\end{array}\right] .
$$

In the Dirac-Pauli representation

$$
\vec{\gamma} \equiv\left[\begin{array}{cc}
0 & -i \vec{\sigma} \\
i \vec{\sigma} & 0
\end{array}\right], \quad \gamma_{4} \equiv\left[\begin{array}{cc}
1 & 0 \\
0 & -1
\end{array}\right]
$$

the equation for a particle takes the form

$$
\left[\begin{array}{cc}
-E+m c^{2} & \hbar c \vec{\sigma} \vec{k} \\
-\hbar c \vec{\sigma} \vec{k} & E+m c^{2}
\end{array}\right]\left[\begin{array}{c}
\varphi(\vec{k}) \\
\chi(\vec{k})
\end{array}\right]=0 .
$$

Hence the expression for the positive energy of the particle:

$$
E=\sqrt{(\hbar c k)^{2}+\left(m c^{2}\right)^{2}}
$$

Since functions (7), (10) are considered for which the Fourier decomposition is carried out only at positive frequencies, a root with a negative sign for energy should not be taken into account. Similar relations hold for antiparticles. Thus, solutions of equations (40) can be written as

$\psi(\vec{k})=\left[\begin{array}{c}\varphi(\vec{k}) \\ \frac{\hbar c \vec{k} \vec{\sigma}}{E+m c^{2}} \varphi(\vec{k})\end{array}\right], \quad \eta(\vec{k})=\left[\begin{array}{c}\zeta(\vec{k}) \\ \frac{\hbar \vec{k} \vec{\sigma}}{E+m c^{2}} \zeta(\vec{k})\end{array}\right]$.

To fulfill the normalization conditions for bispinors $\psi(\vec{k})^{+} \psi(\vec{k})=1$ and $\eta^{+}(\vec{k}) \eta(\vec{k})=1$, the following normalization of spinors is necessary:

$$
\varphi^{+}(\vec{k}) \varphi(\vec{k})=\zeta^{+}(\vec{k}) \zeta(\vec{k})=\frac{1}{2}\left(1+\frac{m c^{2}}{E}\right) .
$$

Thus, the general solution of the Dirac equation for a free particle with momentum $\hbar \vec{k}$ can be represented in one of two forms:

$$
\begin{aligned}
\psi(\vec{x}, t) & =\frac{1}{\sqrt{\bar{V}}}\left[\psi_{+}(\vec{k}) e^{i(\vec{k} \vec{x}-\omega t)}+C^{*} \tilde{\bar{\eta}}_{+}(\vec{k}) e^{-i(\vec{k} \vec{x}-\omega t)}\right], \\
\eta(\vec{x}, t) & =\frac{1}{\sqrt{V}}\left[\eta_{+}(\vec{k}) e^{i(\vec{k} \vec{x}-\omega t)}+C^{*} \tilde{\bar{\psi}}_{+}(\vec{k}) e^{-i(\vec{k} \vec{x}-\omega t)}\right],
\end{aligned}
$$

where $\omega=E / \hbar=\sqrt{(\hbar c k)^{2}+\left(m c^{2}\right)^{2}} / \hbar$ - positive frequency. However, as noted, these functions have no sense of probability amplitudes and cannot be used to calculate probability characteristics. As in the nonrelativistic theory, wave functions (39), which have a sense of probability amplitudes, describe a delocalized particle and an antiparticle with a certain momentum and positive energy. In the general case, the wave functions of particles and antiparticles, decomposed into plane waves, have the form

$$
\begin{aligned}
\psi_{+}(\vec{x}, t) & =\frac{1}{\sqrt{V}} \sum_{k, r} c_{r}(\vec{k}) \psi(\vec{k}, r) e^{i(\vec{k} \vec{x}-\omega t)} \\
\eta_{+}(\vec{x}, t) & =\frac{1}{\sqrt{V}} \sum_{k, r} b_{r}(\vec{k}) \eta(\vec{k}, r) e^{i(\vec{k} \vec{x}-\omega t)}
\end{aligned}
$$

Here the index $r= \pm 1$ numbers the spinors with the projection of the spin on the axis $z$ different in the rest system. For bispinors in (48) the conditions of orthonormality are satisfied

$$
\psi^{+}(\vec{k}, r) \psi\left(\vec{k}, r^{\prime}\right)=\delta_{r r^{\prime}}, \quad \eta^{+}(\vec{k}, r) \eta\left(\vec{k}, r^{\prime}\right)=\delta_{r r^{\prime}} .
$$

For the expansion coefficients in (48), the normalization conditions are also satisfied

$$
\sum_{k, r}\left|c_{r}(\vec{k})\right|^{2}=\sum_{k, r}\left|b_{r}(\vec{k})\right|^{2}=1 .
$$


The conditions for the completeness of the wave functions of a particle and an antiparticle with positive energies can be written in one of the equivalent forms:

$$
\begin{aligned}
& \sum_{r= \pm 1}\left[\psi_{+}(\vec{k}, r) \bar{\psi}_{+}(\vec{k}, r)+C^{*} \tilde{\bar{\eta}}_{+}(-\vec{k}, r) \tilde{\eta}_{+}(-\vec{k}, r) C\right]=1 \\
& \sum_{r= \pm 1}\left[\eta_{+}(\vec{k}, r) \bar{\eta}_{+}(\vec{k}, r)+C^{*} \tilde{\bar{\psi}}_{+}(-\vec{k}, r) \tilde{\psi}_{+}(-\vec{k}, r) C\right]=1 .
\end{aligned}
$$

According to (37), the energy and momentum of an electron and positron are determined by the formulas

$$
\begin{aligned}
W & =\sum_{k, r} E(\vec{k})\left[c_{r}^{*}(\vec{k}) c_{r}(\vec{k})+b_{r}{ }^{*}(\vec{k}) b_{r}(\vec{k})\right], \\
\vec{P} & =\sum_{k, r} \hbar \vec{k}\left[c_{r}{ }^{*}(\vec{k}) c_{r}(\vec{k})+b_{r}{ }^{*}(\vec{k}) b_{r}(\vec{k})\right],
\end{aligned}
$$

where $E(\vec{k})=\sqrt{(\hbar c k)^{2}+\left(m c^{2}\right)^{2}}$. Naturally, in the proposed interpretation, the contribution of states with negative energy to the total energy is absent. Formulas (48) - (53) should be used in the transition to the quantum-field description of electrons and positrons. The Dirac equations describing electrons and positrons in stationary fields have the same form as in the standard approach [2]. The difference is that there is no need to take into account the states with negative energy $E<-m c^{2}$, which do not exist. Therefore, naturally, there is no probability of tunneling into such states, and there are no "paradoxes" due to consideration of such states (various versions of the "Klein paradox" $[2,6])$.

\section{ELECTRONS AND POSITRONS STATES IN NONSTATIONARY ELECTROMAGNETIC FIELD}

It was shown above that the states of electrons and positrons with positive energies in a stationary electromagnetic field are independent, and they are described by the same unrelated equations differing only by the sign of the charge. We consider equations in the presence of a nonstationary field. We write the vector potential in the form of a sum of constant and variable components in time: $A_{\mu}(x)=$ $A_{\mu}(\vec{x})+\underline{A}_{\mu}(x)$. In this case, equation (14) takes the form

$$
Q(x)+C^{*} \tilde{\bar{\Pi}}(x)=i e \underline{A}_{\mu}(x) \gamma_{\mu}\left(\psi_{+}(x)+C^{*} \tilde{\bar{\eta}}_{+}(x)\right),
$$

where $Q(x)$ and $\Pi(x)$ defined by formulas (15). Equation (54) can also be divided into an equation for particles, when $\psi_{+}(x)=0$, and an equation for antiparticles, when :

$$
\begin{array}{r}
\hbar c \gamma_{\mu} \frac{\partial \psi_{+}}{\partial x_{\mu}}-i e A_{\mu}(\vec{x}) \gamma_{\mu} \psi_{+}+m c^{2} \psi_{+}= \\
=-i e \underline{A}_{\mu}(x) \gamma_{\mu} \psi_{+}, \\
\hbar c \gamma_{\mu} \frac{\partial \eta_{+}}{\partial x_{\mu}}+i e A_{\mu}(\vec{x}) \gamma_{\mu} \eta_{+}+m c^{2} \eta_{+}= \\
=-i e \underline{A}_{\mu}(x) \gamma_{\mu} \eta_{+} .
\end{array}
$$

In a nonstationary external field, energy is, of course, not conserved.

\section{CONCLUSIONS}

Usually, when difficulties in the Dirac theory are discussed, it is noted that this theory is "one-particle", i.e. describes one relativistic electron or positron, free or in an external electromagnetic field. Therefore, it is believed that the difficulties encountered by the theory must be overcome in the transition to a quantum-field description based on the secondary quantization apparatus, which is undoubtedly correct. However, as discussed above, many difficulties disappear within the framework of the one-particle theory, if we consistently adhere to the probabilistic interpretation of functions describing the state of a particle. Obviously, not every complex function of coordinates and time can be considered as a probability amplitude. This, in particular, applies to the multicomponent complex field described by the Dirac equation. The general solution of the Dirac equation contains both a positive-frequency part, describing states with positive energies, and a negativefrequency part, which correspond to states with energies of opposite sign. Only states with positive energies have a physical meaning, and the negativefrequency part cannot be directly interpreted as a function describing real states. Thus, the general solution of the Dirac equation cannot be interpreted as a wave function of the physical state. However, it is impossible to simply discard the states with negative energy, since the functions of states with positive energies do not form a complete set. To overcome this contradiction, one should take into account that, as shown by many years of research, the Dirac theory describes two types of particles with identical masses, but opposite signs of charge. Therefore, it is natural, along with the Dirac equation containing a charge of one sign, to simultaneously consider also the Dirac equation with a charge of opposite sign. With these equations, the positive-frequency solutions turn out to be independent, and their unobservable negativefrequency solutions, when using the charge conjugation matrix, are expressed in terms of these positivefrequency solutions. Thus, in the Dirac theory, as a complete set of physical states that have a sense of probability amplitudes, one should take positivefrequency solutions of the Dirac equations with the same mass and with opposite charge signs.

In connection with the review, a question may arise as to why, with a fully charge-symmetric description, only positive-frequency solutions respond to physical states. Where and at what stage does asymmetry arise with respect to functions with a different energy sign? This, as can be seen, is connected with the assumption that states that correspond to a minimum of energy are realized in nature. Otherwise, it would be advisable to select functions that describe states with negative energies as observables. 


\section{References}

1. 1. P.A.M. Dirac. On the creation of quantum field theory: main articles of 1925 - 1958 years. M.: "Nauka", 1990, 368 p.

2. A.I. Akhiezer, V.B. Berestetskii. Quantum electrodynamics. M.: "Nauka", 1981, 432 p.

3. N.N. Bogoliubov, D.V.Shirkov. Introduction to the theory of quantum fields. M.: "Nauka", 1973, $416 \mathrm{p}$.

4. N.N. Bogoliubov, D.V.Shirkov. Quantum fields. Addison-Wesley, 1982, 388 p.

5. S.S. Schweber. An introduction to relativistic quantum field theory. Dover Publications, 2005, $928 \mathrm{p}$.

6. J.D. Bjorken, S.D. Drell. Relativistic quantum mechanics. McGraw-Hill Education, 1998, 314 p.

7. J.D. Bjorken, S.D. Drell. Relativistic quantum fields. Dover Publications, 2014, 416 p.

8. V.B. Berestetskii, L.P. Pitaevskii, E.M. Lifshitz. Quantum electrodynamics. V.4. ButterworthHeinemann, 2012, 667 p.
9. R.P. Feynman. Quantum electrodynamics. Westview Press, 1998, 208 p.

10. S. Weinberg. The quantum theory of fields. v.1. Foundations Cambridge University Press, 2005, $609 \mathrm{p}$.

11. M.E. Peskin, D.V.Schroeder. An introduction to quantum field theory. CRC Press, 1995, $866 \mathrm{p}$.

12. S.M. Bilenky. Introduction to Feynman diagrams. Pergamon Press, 1974, $196 \mathrm{p}$.

13. W.E. Thirring. Principles of quantum electrodynamics. Academic Press, 2013, 250 p.

14. A.A.Sokolov, I.M. Ternov, A.Ch. Zhukovskii, A.V. Borisov. Quantum electrodynamics. M.: "Mir", 1988, 335 p.

15. A.I. Akhiezer, S.B. Peletminskii. Theory of fundamental interactions. World Sci. Publ., 1994, $500 \mathrm{p}$.

16. L.D. Landau, E.M. Lifshitz. Quantum mechanics: non-relativistic theory. Pergamon Press, 1977, $673 \mathrm{p}$.

\section{ЗАРЯДОВО-СИММЕТРИЧНОЕ ОПИСАНИЕ ЭЛЕКТРОНОВ И ПОЗИТРОНОВ В ТЕОРИИ ДИРАКА БЕЗ ОТРИЦАТЕЛЬНЫХ ЭНЕРГИЙ}

\section{Ю. М. Полуэктов}

Предложена симметричная относительно частиц и античастиц формулировка теории Дирака, в которой отсутствуют состояния с отрицательной энергией. Полям частиц и античастиц сопоставлены волновые функции, для которых справедлива борновская интерпретация как амплитуд вероятности. Тем самым в теории устраняются различные "парадоксы", существование которых обусловлено некорректным учётом состояний с отрицательной энергией.

\section{ЗАРЯДОВО-СИМЕТРИЧНИЙ ОПИС ЕЛЕКТРОНІВ ТА ПОЗИТРОНІВ У ТЕОРІЇ ДІРАКА БЕЗ ВІД'ЄМНИХ ЕНЕРГІЙ}

\section{Ю. М. Полуектов}

Запропоновано симетричне щодо частинок і античастинок формулювання теорії Дірака, в якій відсутні стани з від'ємною енергією. Полям частинок і античастинок зіставлені хвильові функції, для яких справедлива борнівська інтерпретація як амплітуд імовірності. Тим самим у теорії усуваються різноманітні "парадокси", існування яких обумовлено некоректним урахуванням станів 3 від'ємною енергією. 